\title{
A novel double deletion underscores the importance of characterizing end points of the CFTR large rearrangements
}

\author{
Magali Taulan ${ }^{\star 1,2,3}$, Caroline Guittard ${ }^{2,3}$, Corinne Theze ${ }^{2,3}$, Mireille Claustres ${ }^{1,2,3}$ \\ and Marie des Georges ${ }^{2,3}$
}

\author{
${ }^{1}$ Université Montpellier1, UFR de Médecine, Montpellier, France ${ }^{2}$ INSERM U827, Laboratoire de Génétique de Maladies \\ Rares, Montpellier, France; ${ }^{3}$ CHU Montpellier, Hôpital Arnaud de Villeneuve, Laboratoire de Génétique Moléculaire, \\ Montpellier, France
}

Large genomic rearrangements in patients with cystic fibrosis (CF) account for up to $16-24 \%$ of CF alleles negative for point mutations in European populations. Herein, we identified a new large rearrangement removing exon 19 in a young CF patient, who hitherto harbored only the F508del mutation. By using LightCycler technology, we successfully and rapidly delineated the deletion end points by determining the relative copy number of a set CFTR sequence from introns 18 to 19. Fine mapping of the sequences bordering its break points was achieved using direct sequencing. We reported the first complex CFTR rearrangement containing two successive deletion events putatively linked. We evidenced the presence of short direct repeats in the vicinity of the deletions suggesting a possible replication slippage model. In this report, we also discussed the putative molecular mechanism and consequences of this complex gene rearrangement, unprecedented in CF. This complex deletion illustrates the importance of delineating the genomic rearrangement to improve our knowledge of the CFTR mutational spectrum and to better understand the molecular mechanism controlling the CFTR expression.

European Journal of Human Genetics (2009) 17, 1683-1687; doi:10.1038/ejhg.2009.73; published online 13 May 2009

Keywords: cystic fibrosis; CFTR gene; genomic rearrangement; large deletion

\section{Introduction}

Although short deletions and insertions are frequent, the implications of gross genomic rearrangements in cystic fibrosis (CF) are now well documented. ${ }^{1-3}$ In CF, the most common lethal autosomal recessive disease in Caucasians, $\sim 2 \%$ of CF mutations, correspond to large deletions, insertions or duplications. Half of these mutations are a simple deletion from one to the whole cystic fibrosis

*Correspondence: $\operatorname{Dr} M$ Taulan, Université Montpellier1, UFR de Médecine, INSERM U827, Laboratoire de Génétique de Maladies Rares, CHU, 641 avenue du Doyen Gaston Giraud, Montpellier F-34000, France. Tel: (33) 4674153 64; Fax: (33) 467415365 ;

E-mail: magali.taulan@inserm.fr

Received 2 February 2009; revised 2 April 2009; accepted 8 April 2009; published online 13 May 2009 transmembrane conductance regulator (CFTR) gene or complex deletion/insertions (www.genet.sickkids.on.ca/cftr/app). Only two complex deletions have hitherto been identified, including a deletion of $50 \mathrm{~kb}$ removing exons $4-7$ and $11-$ $18^{4}$ and the CFTRdele $3-10,14 \mathrm{~b}-16 .^{5}$ This report focused on the identification of a novel complex gene rearrangement with a deletion of exon 19 in a young CF patient carrying the paternal F508del allele. Another investigation has evoked a deletion of exon 19 in a CF patient, starting $1.5 \mathrm{~kb}$ after the last nucleotide of exon 18 and spanning 5279 bp. However, the break point junctions are unsimilar. ${ }^{6}$ Here, we presented our successful approach to accurately map the position of the break points. As the use of long-range PCR did not always allow obtaining of results, we next developed a simple and rapid test to refine the deletion end points by using quantitative PCR 
technology. We also proposed a putative model to explain this double deletion and discussed the potential consequences of exon 19 disruption.

\section{Methods}

\section{CF patient}

This report focused on a young patient who carried the F508del mutation inherited from her father; the second mutation remained unknown after complete sequencing of the CFTR gene. She presented a classical form of CF. Informed consent to CFTR studies had been obtained earlier from parents at the time of referral.

Search of gross rearrangements in the CFTR gene by semiquantitative fluorescent PCR (SQF-PCR) analysis To detect large exon deletions and duplications in the CFTR gene, we carried out a robust SQF-PCR assay developed in our laboratory as reported earlier (primers on demand). ${ }^{2}$

Confirmation of deleted exon and determination of the break point junctions by quantitative PCR (qPCR) assays

To identify the end-point junctions, we carried out a set of amplifications by using LightCycler 480 technology according to the manufacturer's recommendations
(Roche Diagnostics, Meylan, France). We performed the relative quantification of several sequences from introns 18 to 19. As reference internal control, amplification of $\beta$-actin gene (Eurogente, Angers, France) was carried out following the manufacturer's recommendations. PCRs were set up in triplicate in a total volume of $10 \mu \mathrm{l}$ containing $5 \mu \mathrm{l}$ mix Sybr Green, $20 \mathrm{ng}$ DNA and $1 \mu \mathrm{l}$ mix primers $(5 \mu \mathrm{M}$ final). By using the LightCycler Probe Design Software 2.0, primer pairs (amplicon of 150-200 bp) were designed every $2000 \mathrm{bp}$ on a genomic region encompassing exon 19 (Table 1). The copy number of each part amplified from introns 18 to19 was evaluated.

The exact delineation of the deletion was carried out by directsequencing using primers flanking the deletion (noted in bold in Table 1).

\section{Familial segregation and epidemiological studies}

The presence of the small deletion in the patient and her family was also verified by carrying out a simple PCR using primers Del37 bp (Table 1). Amplification products were separated by electrophoresis on a $2 \%$ agarose gel and visualized by ethidium bromide staining.

To screen the frequency of the small deletion ( $37 \mathrm{bp}$ ), we carried out an epidemiological study in a panel of 190 chromosomes from the control population, 26 from $\mathrm{CF}$ patients with only one mutation detected, 90 from

Table 1 List of primers used for delineating the exon 19 deletion

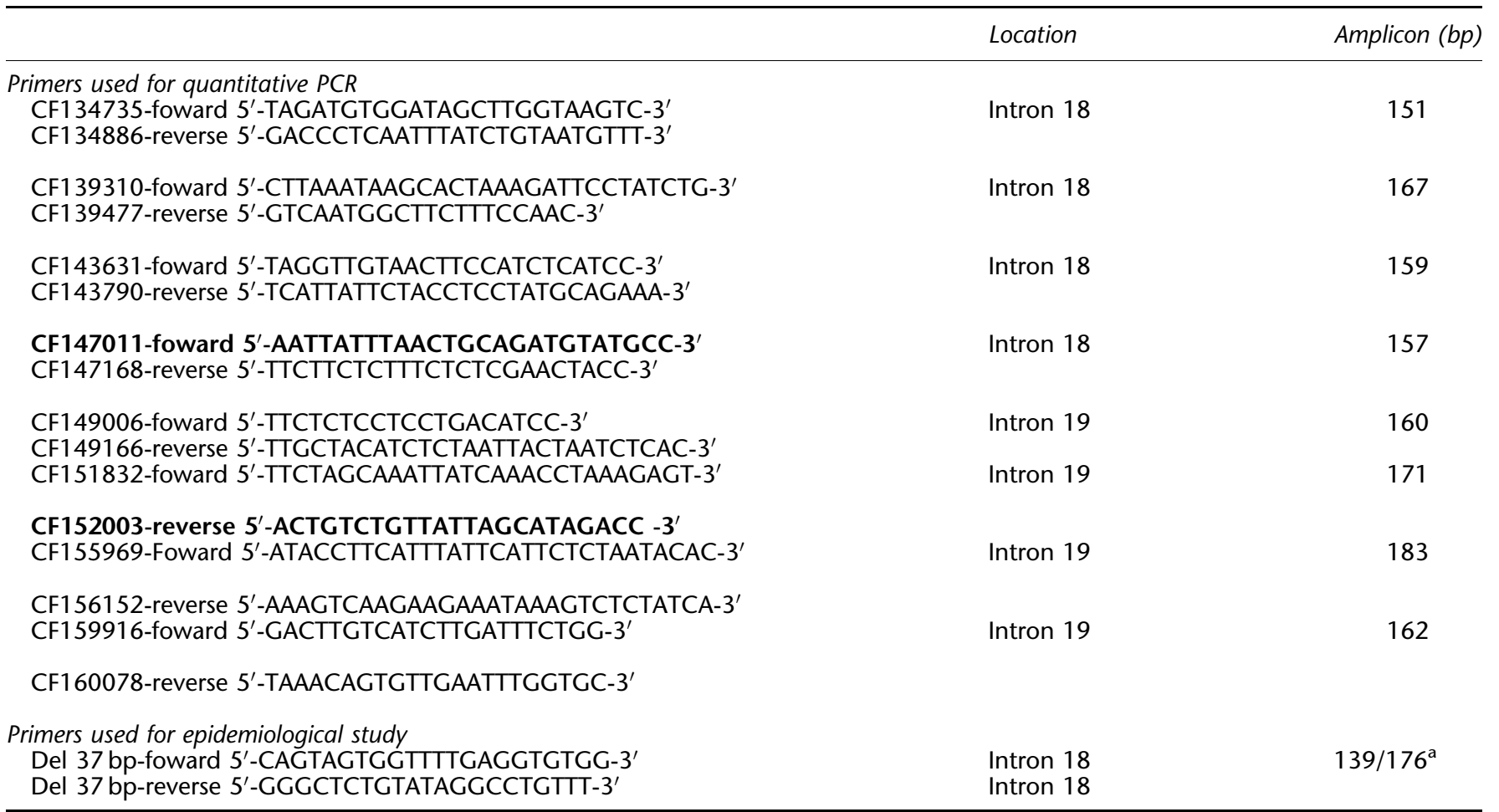

${ }^{a}$ size of the amplicon in bp obtain in patient/in control.

Primers in bold are used for sequencing assays. 
congenital bilateral absence of the vas deferens (CBAVD) with either none or one mutation identified and 44 from patients homozygous for F508del.

\section{Nomenclature of mutations}

For the convenience of readers, we used the usual mutation nomenclature reported to the International Consortium Mutation Database (http://www.genet.sickkids.on.ca/cftr/app) in accordance with the CFTR gene numbering (GenBank NM_00492.3) with the A of the ATG translation start codon numbered +133 . However, for the exon 19 deletion, the international nomenclature recommended in the Human Genome Variation Society web page (http://www.hgvs.org/ mutnomen/) was also indicated (in italic and in brackets).

\section{Results}

Identification of the exon 19 deletion and delineation of break-point junctions

By using SQF-PCR, we detected a deletion of exon 19 in both the patient's and mother's DNA. We next developed a simple and rapid test to confirm the identified deletion and refine the end points using quantitative PCR technology. We evaluated the relative copy number of a set of CFTR sequences from introns 18 to 19 (Table 1). The copy number of exon 19 was twofold lower in both the patient's and mother's DNA than in the control DNA, confirming the presence of a deletion.

To precisely map the junction fragments of the exon 19 deletion, we carried out direct sequencing assays using primers designed for the qPCR tests and flanking the deletion (Table 1). This rearrangement consists of a small deletion of $37 \mathrm{bp}$ located in intron 18, a conserved sequence of $103 \mathrm{bp}$ and a gross deletion of $4260 \mathrm{bp}$ spanning exon 19 (Figure 1a). This deletion can be written thus: 3601-331_3601-294del37; 3601189_3849+3822del4260 bp (c.3469-331_c.3469-294del37; c.3469-189_c.3717+3822del4260 bp).

Familial segregation was performed to verify whether the mother harbored the small $37 \mathrm{bp}$ deletion. As observed in Figure $1 \mathrm{~b}$, we amplified a 139-bp fragment only when the deletion was present, whereas the 176-bp products correspond to the amplification control. This small intronic deletion has been evidenced only in the patient and in her mother (Figure 1b). This result evidenced that both deletions are transmitted in cis. To evaluate whether this double deletion results from two independent events in close proximity, an epidemiological study was carried out

a

Deletion of $37 \mathrm{bp}$

Deletion of 4,260 bp

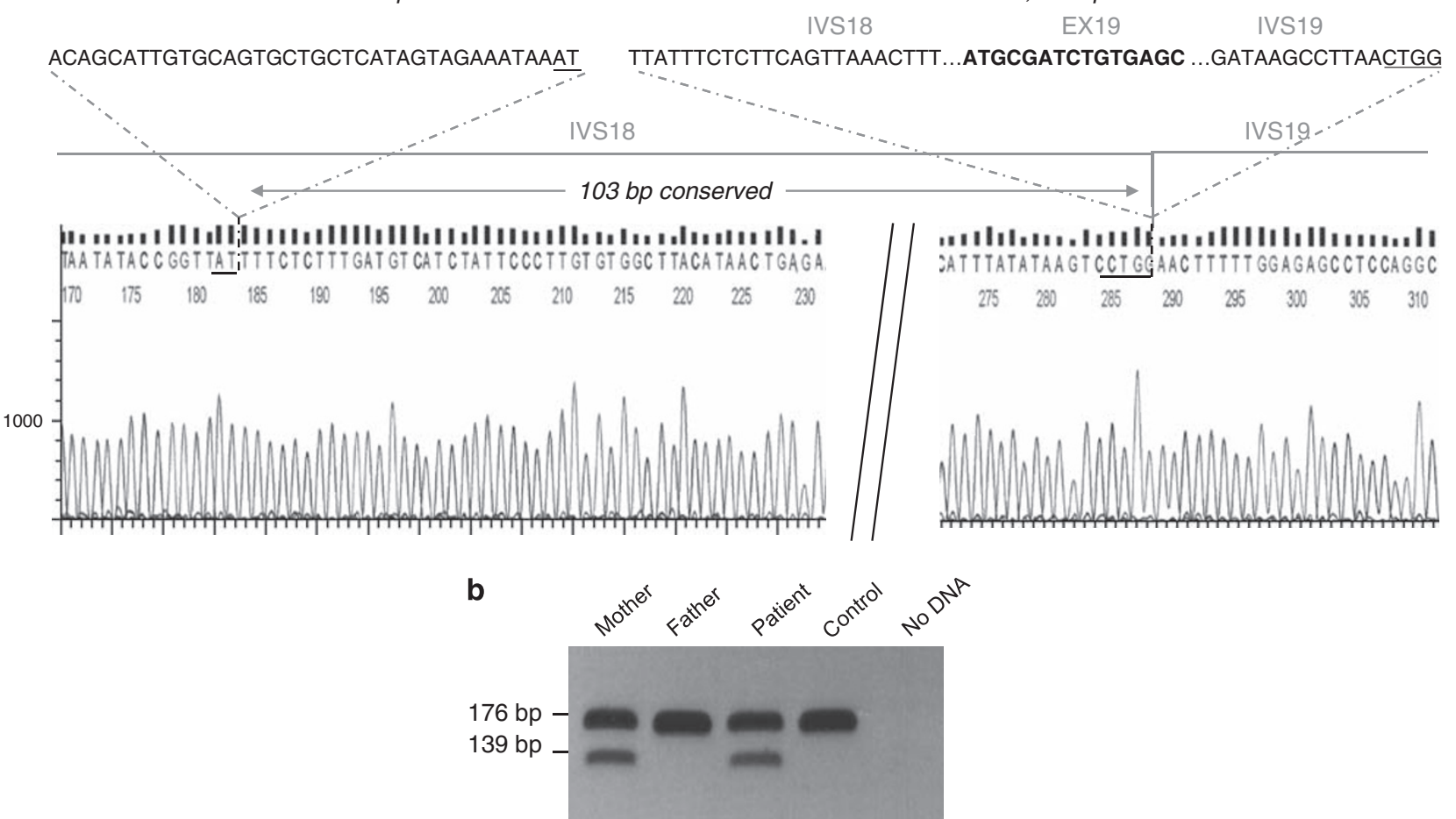

Figure 1 Confirmation of a double deletion c.3469-331_c.3469-294del37; c.3469-189_c.3717+3822del4260 bp in cystic fibrosis. (a) The breakpoint junctions of both deletions and conserved sequences were determined by direct sequencing. This rearrangement consists of a small deletion of $37 \mathrm{bp}$ and a gross deletion of $4260 \mathrm{bp}$ spanning exon 19 and a conservation of $103 \mathrm{bp}$. (b) Amplification reactions confirmed the presence of the small deletion in the patient and the mother, indicated by specific PCR products on $2 \%$ agarose gels by comparison with the non-deleted allele (control). 
in a panel of 190 chromosomes from the control population and 160 from either CF or CBAVD patients. To date, the small deletion has been identified only in the patient who inherited it from her mother.

\section{Short direct repeats at the end points of the exon 19 deletion}

To explain the putative molecular mechanism involved in the generation of both deletion events, we used mfold software (http://mobyle.pasteur.fr/cgi-bin/MobylePortal/ portal.py?form $=$ mfold) that predicts the secondary structure of single-stranded nucleic acids. It revealed a hairpin structure potentially formed by the direct TA repeats resulting in the small deletion (Figure 2, loop a, left representation). We also proposed another putative looped secondary structure beginning with the two inverted repeats and with 16 out of 37 nucleotides predicted to be paired (Figure 2, loop a, right representation). Inspection of the sequence across the large 4260 bp deletion showed the presence of 4-bp direct repeats CTGG (Figure 2, loop b). Computer analysis of the sequence internal to two direct repeats in the deletion revealed a potential looped secondary structure near the $5^{\prime}$ and $3^{\prime}$ deletion break points.

These hairpin structures were supposed to promote a forward slippage resulting in a deletion.

\section{Discussion}

In this study, we reported the mapping of a complex rearrangement involving two discontinuous deletions removing exon 19 in a young CF patient carrying F508del on the paternal chromosome. This mutation corresponds to a double deletion removing $37 \mathrm{bp}$ and $4260 \mathrm{bp}$ with a conserved sequence of $103 \mathrm{bp}$. Although two other CFTR complex deletions resulting in two genomic disruptions have been reported, ${ }^{4,5}$ the two deletion events are not in close proximity. Earlier investigations on other genes identified large rearrangements in which the intervening motif between the two successive deletions represents a small sequence from 7 to 167 bp. ${ }^{7,8,9,10}$ However, we cannot rule out the possibility that this double deletion corresponds to two separate mutational events. The $37 \mathrm{bp}$ deletion alone might result in an intronic sequence inclusion involving the creation of a novel exon introducing a frameshift as reported earlier. ${ }^{11}$ To ascertain that this mutation did not correspond to an unidentified mutation, we carried out a scanning analysis and showed that this small deletion was absent in health controls from the same population. These data strongly suggest that our complex deletion occurred as intrinsically linked events. By using the 'exon-phasing tool' of the UMD software (Universal Mutation Database, http:// www.umd.be), ${ }^{12}$ the double deletion described in this report potentially causes an in-frame deletion of exon 19 .

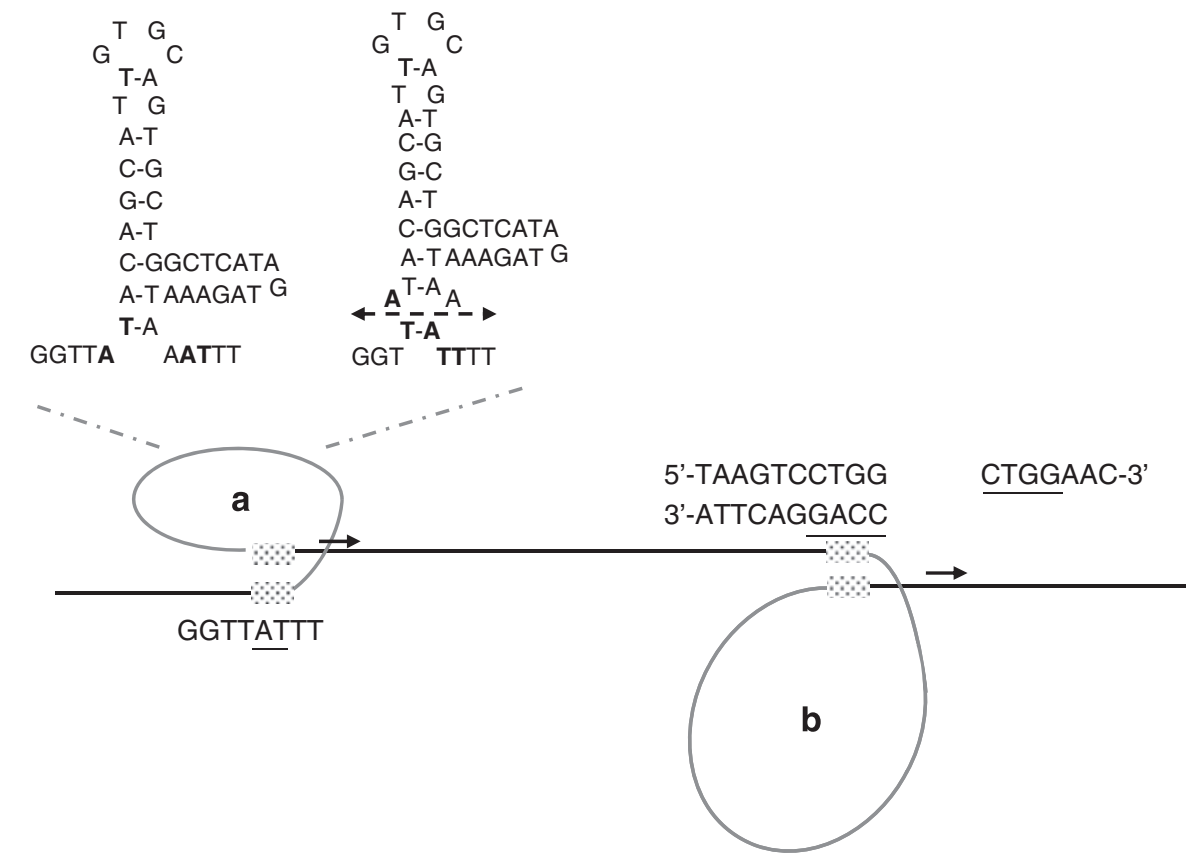

Figure 2 Mechanism proposed for the formation of both deletions. For the small deletion (loop a), putative hairpin structures generated by either direct TA repeats (noted in bold, left representation) or inverted repeats (noted in bold, right representation) are presented. The double arrow represents the putative break site. For the large deletion (loop b), we presented the direct 4-bp repeats that might promote the splippage mechanism. The horizontal arrows indicate the direction of DNA replication. 
The resulting putative CFTR protein lacks 82 amino acids and thus part of the second nucleotide-binding domain (NBD2), which is involved in CFTR gating by ATP hydrolysis ${ }^{13}$ and seems critical for CFTR function.

The presence of direct repeats at the end points of both deletions are now well documented to facilitate the slippage model during replication process. ${ }^{14,15}$ These include consecutive forward replication slippages in cis generating a double deletion as depicted in Figure 2. Indeed, the presence of short sequence homologies $(2-8 \mathrm{bp})$ at the junction fragments has been evoked in non-homologous recombinations, such as slippage ${ }^{15}$ and mispairing of elements homology during DNA replication. Comparative analysis of several characterized deletions showed locus variability both in the sequence and length of the direct repeats, suggesting that other factors, such as chromatin structure and chromosomal location of the genetic locus, may play an important role in generating rearrangements. Interestingly, Phylactides et $a l^{16}$ evidenced that DNAse hypersensitive sites influencing the CFTR gene expression are present in intron 18.

For all that, this study reaffirms the view that large CFTR deletions are an important cause of CF disease. Additional experiments might allow the provision of essential information on the molecular consequences of these mutations to improve our knowledge of phenotype-genotype correlations and also help geneticists in routine practice to classify the gross CFTR genomic rearrangement.

\section{References}

1 Schneider M, Hirt C, Casaulta C et al: Large deletions in the CFTR gene: clinics and genetics in Swiss patients with CF. Clin Genet 2007; 72: 30-38.

2 Taulan M, Girardet A, Guittard C et al: Large genomic rearrangements in the CFTR gene contribute to CBAVD. BMC Med Genet 2007; 8: 22.

3 Paracchini V, Seia M, Coviello D et al: Molecular and clinical features associated with CFTR gene rearrangements in Italian population: identification of a new duplication and recurrent deletions. Clin Genet 2008; 73: 346-352.

4 Morral $\mathrm{N}$, Nunes $\mathrm{V}$, Casals $\mathrm{T}$ et al: Uniparental inheritance of microsatellite alleles of the cystic fibrosis gene (CFTR): identification of a 50 kilobase deletion. Hum Mol Genet 1993; 2: $677-681$.

5 Niel F, Legendre $M$, Bienvenu $T$ et al: A new large CFTR rearrangement illustrates the importance of searching for complex alleles. Hum Mutat 2006; 27: 716-717.

6 Costes B, Girodon E, Vidaud D et al: Prenatal detection by realtime quantitative PCR and characterization of a new CFTR deletion, 3600+15kbdel5.3 kb (or CFTRdele19). Clin Chem 2000; 46: $1417-1420$.

7 Tvrdik T, Marcus S, Hou SM et al: Molecular characterization of two deletion events involving Alu-sequences, one novel base substitution and two tentative hotspot mutations in the hypoxanthine phosphoribosyltransferase (HPRT) gene in five patients with Lesch-Nyhan syndrome. Hum Genet 1998; 103: 311-318.

8 Marlin S, Blanchard S, Slim R et al: Townes-Brocks syndrome: detection of a SALL1 mutation hot spot and evidence for a position effect in one patient. Hum Mutat 1999; 14: 377-386.

9 Buyse IM, Fang P, Hoon KT et al: Diagnostic testing for Rett syndrome by DHPLC and direct sequencing analysis of the MECP2 gene: identification of several novel mutations and polymorphisms. Am J Hum Genet 2000; 67: 1428-1436.

10 Morisawa T, Yagi M, Surono A et al: Novel double-deletion mutations of the OFD1 gene creating multiple novel transcripts. Hum Genet 2004; 115: 97-103.

11 Beck S, Penque D, Garcia S et al: Cystic fibrosis patients with the 3272-26A - > G mutation have mild disease, leaky alternative mRNA splicing, and CFTR protein at the cell membrane. Hum Mutat 1999; 14: 133-144.

12 Beroud C, Hamroun D, Collod-Beroud G et al: UMD (Universal Mutation Database): 2005 update. Hum Mutat 2005; 26: 184-191.

13 Zerhusen B, Ma J: Function of the second nucleotide-binding fold in the CFTR chloride channel. FEBS Lett 1999; 459: 177-185.

14 Chen JM, Chuzhanova N, Stenson PD et al: Meta-analysis of gross insertions causing human genetic disease: novel mutational mechanisms and the role of replication slippage. Hum Mutat 2005; 25: 207-221.

15 Chen JM, Chuzhanova N, Stenson PD et al: Complex gene rearrangements caused by serial replication slippage. Hum Mutat 2005; 26: 125-134.

16 Phylactides M, Rowntree $\mathrm{R}$, Nuthall $\mathrm{H}$ et al: Evaluation of potential regulatory elements identified as DNase I hypersensitive sites in the CFTR gene. Eur J Biochem 2002; 269: 553-559. 